\title{
BINARY VARIATIONAL FILTERING FOR TARGET TRACKING IN SENSOR NETWORKS
}

\author{
Jing Teng, Hichem Snoussi, Cédric Richard \\ ICD-LM2S, University of Technology of Troyes \\ 12 rue Marie Curie, BP 2060, 10010 Troyes cedex \\ Phone: +33.3.25.71.58.88 - Fax: +33.3.25.71.56.99 \\ E-mail: jing.teng@utt.fr \\ Url: http://www.cedric-richard.fr
}

\begin{abstract}
Target tracking in wireless sensor networks (WSN) has brought up new practical problems. The limited energy supply and bandwidth of WSN have put stringent constraints on the complexity and inter-node information exchange of the tracking algorithm. In this paper, we propose a binary variational algorithm outperforming existing target tracking algorithms such as Kalman and Particle filtering. The variational formulation allows an implicit compression of the exchanged statistics between leader nodes, enabling thus a distributed decision-making. Its binary extension further reduces the resource consumption by locally exchanging only few bits.
\end{abstract}

\section{INTRODUCTION}

Target tracking problems in wireless sensor networks (WSN) have attracted the attention of many researchers [1, 2, 3, 4]. The sensor network constraints inspire designers to achieve reasonably accurate estimation whilst minimizing overhead of networks configuration and maximizing networks lifetime by managing the energy consumption of the sensors and reducing the networks communication.

Recently, distributed particle filters were proposed in literature [5, 3]. In [3], a message approximating scheme based on a greedy KD-tree approximation is proposed. In [5], a full collaborative strategy is proposed with a GMM (Gaussian Mixture Model) message approximation. The main drawback of these methods is the error propagation through the network when approximating the particle representation by a few number of Gaussian statistics. A quantized centralized particle filter is proposed in [6]. According to this method, the nodes only send a connectivity and a change in proximity information. Recently, a variational approach suitable to the communication constraints of sensor networks has been proposed in [7]. The efficiency of the variational approximation relies on the fact that the online update of the filtering distribution and its compression are simultaneously performed. In addition, the variational approach has the nice property to be parameterization-independent ensuring the robustness of the data processing.

In this contribution, we propose a novel algorithm based on the variational approximation and the connectivity measure. The measured energy on the sensor is quantified according to a fixed threshold, indicating the presence of the target in the vicinity of the node. At each instant, only a few nodes which have detected the target send one bit to a selected leader node to perform target tracking. The leader selection is based on the relevance of nodes positions with respect to the predicted target position.

The paper is organized as follows. In section 2, we briefly recall the SOI-KF filter algorithm (Sign of InnovationsKalman Filtering [8]) and the particle filter in tertiary wireless sensor networks [6]. Section 3 is devoted to the main contribution of this paper, where we propose a distributed binary variational tracking (BVF) algorithm. In section 4, simulation results corroborate the effectiveness and the robustness of the proposed algorithm in difficult tracking situations such as abrupt changes in target trajectory.

\section{RELATED WORK}

\subsection{SOI-KF}

The principal contribution of the SOI-KF algorithm [8] is the quantization function which severely quantize the observation of each sensor to $1 \mathrm{bit}$. The message communicated $b(n)$ is defined as the sign of innovation (SOI):

$$
\begin{aligned}
b(n) & =\operatorname{sign}[y(n)-\hat{y}(n \mid n-1)] \\
& := \begin{cases}+1 & \text { if } y(n) \geq \hat{y}(n \mid n-1) \\
-1 & \text { if } y(n)<\hat{y}(n \mid n-1)\end{cases}
\end{aligned}
$$

where $y(n)$ is the current observation of the $n$th sensor, and $\hat{y}(n \mid n-1)$ is the data prediction of it based on past observation. Relying on the sequence $\mathbf{b}_{0: n}:=[b(0), \ldots, b(n)]^{T}$, an approximate MMSE estimation of target position is constituted. The SOI-KF is however implemented in a centralized way and relies on measuring ranging information from signal 
strength which requires hardware that is typically not available on sensor nodes. In addition, because of the linearity of the state equation, the filtering algorithm is not able to track abrupt changes in the target trajectory.

\subsection{Particle Filtering in Tertiary WSN}

The likelihood models used in target tracking are often nonlinear functions of the state, which result in non-Gaussian posterior distributions. The tracking recursion is thus analytically intractable and non- parametric methods such as Particle Filtering [9] allow a more attractive alternative to KF in providing numerical approximation to track complex models.

The principle idea of tertiary WSN [6] is similar to that of SOI-KF to some extent. In both of them, sensors transmit only one bit during the target tracking process. But unlike SOI-KF, the sensors do not estimate the target's position and can not measure the exact distances between the target and themselves. In brief, tertiary WSN are composed of sensors that emit information based on the strength of the sensed signal in consecutive instants, that is, the $i$-th sensor $(i=1, \ldots, N)$ received signal at instant $t$. If the sensed signal $y_{i, t}>\gamma$, where $\gamma$ is a threshold of the sensor, the transmitted signal is generated according to

$$
s_{i, t}= \begin{cases}+1 & \text { if } y_{i, t}-y_{i, t-1} \geq 0 \text { and } y_{i, t}>\gamma \\ -1 & \text { if } y_{i, t}-y_{i, t-1}<0 \text { and } y_{i, t}>\gamma\end{cases}
$$

So $s_{i, t}=+1$ means that the target is in the vicinity of the $i$-th sensor and is approaching it as well; the "-1" means that the target is receding from the sensor but still within its sensing range. If $y_{i, t}<\gamma$, the sensor does not emit anything (which is considered to be 0 in the computation phase). So it provides highly compressed information about the moving target but retains its direction and proximity information. Such design minimizes the power needed for operation in the sensors as well as the communication bandwidth needed for signal transmission.

Even though the algorithm estimates the target trajectory and its velocity at low cost, the proposed approach in [6] relies on a linear state equation, reducing its ability to track abrupt trajectory changes. Besides, all the information is transmitted to and then processed in the central unit, which will definitely aggravate the burden of the central unit.

\section{BINARY VARIATIONAL FILTERING}

In a distributed context, the variational tracking compresses the message between leader nodes in a consistent manner [7]. The approximated filtering distribution is updated at each instant in a variational approach allowing the covering of the whole state space. In other words, the update of the filtering distribution and its approximation are jointly performed. Thus distributed signal processing is achieved effectively. By using the observations of the distances between the target and its three nearest sensors, accurate results are achieved in [7]. However, adding the required hardware for measuring distances increases the cost and size of the nodes. This motivates the use of a binary quantization process where each node emits one bit stating if the target is in its vicinity or not. An other key point of the variational approach is the use of a general state model [10] to describe the state evolvement instead of the kinematic parameter models applied in [6]; Thirdly, the sensors randomly scatter on the field and only the nearest sensors that sensed the target transmit their observations to the local leader node, which greatly cuts down the cost of communication.

\subsection{General State Model}

The hidden state $x_{t}$ is assumed to be Gaussian distributed, with a random mean $\mu_{t}$ and a random precision matrix $\lambda_{t}$ to further capture the uncertainty about the state distribution:

$$
\left\{\begin{aligned}
x_{t} & \sim \mathcal{N}\left(x_{t} \mid \mu_{t}, \lambda_{t}\right) \\
\mu_{t} & \sim \mathcal{N}\left(\mu_{t} \mid \mu_{t-1}, \bar{\lambda}\right) \\
\lambda_{t} & \sim \mathcal{W}_{\bar{n}}\left(\lambda_{t} \mid \bar{S}\right)
\end{aligned}\right.
$$

Where $\bar{\lambda}$ is a fixed precision matrix to reflect the region of uncertainty for the new estimation around the old one. The state precision matrix is modeled by a Wishart distributed with $\bar{n}$ and $\bar{S}$ denoting respectively the degrees of freedom and precision matrix, both assumed to be fixed.

\subsection{Variational Tracking Algorithm}

Given the model above, the distribution of interest for tracking is the posterior $p\left(\boldsymbol{\alpha}_{t} \mid \boldsymbol{y}_{1: t}\right)$, where $\boldsymbol{\alpha}_{t}=\left(x_{t}, \mu_{t}, \lambda_{t}\right)$ denotes the extended hidden state. It is approximated by a distribution $q\left(\boldsymbol{\alpha}_{t}\right)$ that minimizes the Kullback-Leibler (KL) divergence error. Supposing that $q\left(\boldsymbol{\alpha}_{t}\right)=\prod_{i} q\left(\alpha_{i}\right)$, where $\left\{\alpha_{i}\right\}$ denotes the subsets of $\boldsymbol{\alpha}_{t}$ that are $x_{t}, \mu_{t}, \lambda_{t}, q\left(\boldsymbol{\alpha}_{t}\right)$ is of the form,

$$
q\left(\alpha_{i}\right) \propto \exp <\log p\left(\boldsymbol{y}_{1: t}, \boldsymbol{\alpha}_{t}\right)>_{\prod_{j \neq i} q\left(\alpha_{j}\right)}
$$

where $<.>_{q}$ denotes the expectation operator relative to the distribution $q$.

Taking into account the separable approximate distribution at time $t-1$, the filtering distribution at time $t$ is written,

$$
\begin{aligned}
& p\left(\boldsymbol{\alpha}_{t} \mid \boldsymbol{y}_{1: t}\right) \propto p\left(y_{t} \mid x_{t}\right) p\left(x_{t} \mid \mu_{t}, \lambda_{t}\right) p\left(\lambda_{t}\right) q_{p}\left(\mu_{t}\right), \\
& \text { with } q_{p}\left(\mu_{t}\right)=\int p\left(\mu_{t} \mid \mu_{t-1}\right) q\left(\mu_{t-1}\right) d \mu_{t-1} .
\end{aligned}
$$

The temporal dependence on the past is hence reduced to the incorporation of only one component approximation $q\left(\mu_{t-1}\right)$. The density $q\left(\mu_{t-1}\right)$ turns out to be a Gaussian distribution, limiting the communication between two successive leaders to only sending the mean and the covariance of it. The approximate distribution yields thus a natural and adaptive compression of the filtering distribution which is propagated in the sensor network without lossy compression. 


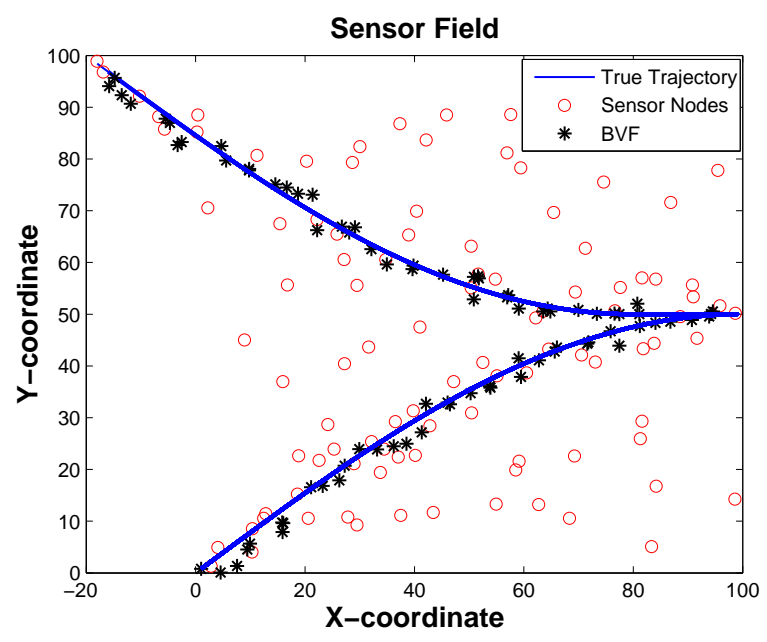

Fig. 1. Binary Variational Filtering (BVF)

Taking into account the prior mean-scale mixture transition model, the updated separable distribution $q\left(\boldsymbol{\alpha}_{t}\right)$ has the following form:

$$
\begin{aligned}
q\left(x_{t}\right) & \propto p\left(y_{t} \mid x_{t}\right) \mathcal{N}\left(x_{t} \mid\left\langle\mu_{t}\right\rangle,\left\langle\lambda_{t}\right\rangle\right) \\
q\left(\mu_{t}\right) & \propto \mathcal{N}\left(\mu_{t} \mid \mu_{t}^{*}, \lambda_{t}^{*}\right) \\
q\left(\lambda_{t}\right) & \propto \mathcal{W}_{n^{*}}\left(\lambda_{t} \mid \boldsymbol{S}_{t}^{*}\right)
\end{aligned}
$$

where the parameters are iteratively updated according to the following scheme:

$$
\begin{aligned}
\mu_{t}^{*} & =\lambda_{t}^{*-1}\left(\left\langle\lambda_{t}\right\rangle\left\langle x_{t}\right\rangle+\lambda_{t}^{p} \mu_{t}^{p}\right) \\
\lambda_{t}^{*} & =\left\langle\lambda_{t}\right\rangle+\lambda_{t}^{p} \\
n^{*} & =\bar{n}+1 \\
\boldsymbol{S}_{t}^{*} & =\left(\left\langle x_{t} x_{t}^{T}\right\rangle-\left\langle x_{t}\right\rangle\left\langle\mu_{t}\right\rangle^{T}-\left\langle\mu_{t}\right\rangle\left\langle x_{t}\right\rangle^{T}+\left\langle\mu_{t} \mu_{t}^{T}\right\rangle+\overline{\boldsymbol{S}}^{-1}\right)^{-1} \\
\mu_{t}^{p} & =\mu_{t-1}^{*} \\
\lambda_{t}^{p} & =\left(\lambda_{t-1}^{*-1}+\bar{\lambda}^{-1}\right)^{-1}
\end{aligned}
$$

Note that the mean state and the precision matrix have closed forms such that their means are easily derived:

$$
\left\langle\mu_{t}\right\rangle=\mu_{t}^{*},\left\langle\lambda_{t}\right\rangle=n^{*} \boldsymbol{S}_{t}^{*},\left\langle\mu_{t} \mu_{t}^{T}\right\rangle=\lambda_{t}^{*-1}+\mu_{t}^{*} \mu_{t}^{* T} .
$$

However, the state $x_{t}$ does not have a closed form approximate distribution. In order to compute its mean and covariance, one can resort to an importance sampling scheme where samples are drawn from the Gaussian $\mathcal{N}\left(x_{t} \mid\left\langle\mu_{t}\right\rangle,\left\langle\lambda_{t}\right\rangle\right)$ and weighted according to their likelihoods (taking into account the binary quantization):

$$
x_{t}^{(i)} \sim \mathcal{N}\left(x_{t} \mid\left\langle\mu_{t}\right\rangle,\left\langle\lambda_{t}\right\rangle\right), w_{t}^{(i)} \propto \prod_{j=1}^{m} p\left(z_{j, t} \mid x_{t}^{(i)}\right),
$$

where $m$ is the number of sensors, and $z_{j, t}$ is the observation transmitted to leader node by the $j$-th sensor at instant $t$. The

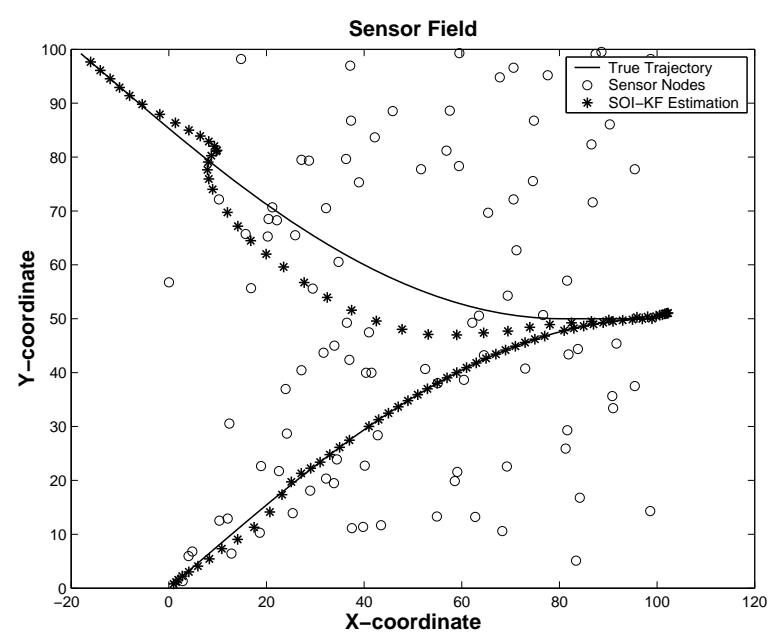

Fig. 2. SOI-KF estimation

distribution $p\left(z_{j, t} \mid s_{j, t}\right)$ is supposed to be $\mathcal{N}\left(\beta_{j} s_{j, t}, \sigma_{\epsilon}^{2}\right), \beta_{j}$ is the attenuation coefficient associated with the $j$-th sensor, and $\sigma_{\epsilon}^{2}$ the noise parameter. $s_{j, t}$ is the connectivity measurement defined as follows:

$$
s_{j, t}= \begin{cases}1, & \text { if } y_{j, t}>\gamma \\ 0, & \text { otherwise }\end{cases}
$$

Note that only the sensors which detected presence of the target transfer proximity information to the leader node.

The mean and covariance are then obtained by their empirical approximations:

$$
\left\langle x_{t}\right\rangle=\sum_{i=1}^{N} w_{t}^{(i)} x_{t}^{(i)},\left\langle x_{t} x_{t}^{T}\right\rangle=\sum_{i=1}^{N} w_{t}^{(i)} x_{t}^{(i)} x_{t}^{(i) T}
$$

To reach the convergence of transition parameters $\left(\mu_{t}^{p}\right.$ and $\left.\lambda_{t}^{p}\right)$, a lot of iterations are involved in BVF. Thus the computation complexity of BVF is much more than that of PF. Thanks to the fast growing of processor development, the computation problem can be resolved easily. On the other side, the main energy expenditure in WSN lies in the communication phase. BVF dramatically reduces the number of bits transmitted between the sensors and also that of leaders without accuracy loss, cutting down the hardware configuration of sensing module at the same time.

\section{SIMULATION RESULTS}

To demonstrate the effectiveness of the proposed Binary Variational Filtering (BVF) algorithm, we have considered the target tracking of a fixed trajectory during 100 time slots, where an abrupt change occurred at instant 51 ( the target jumps from position $[100,50]$ to $[80,50])$. A set of 100 sensors are uniformly randomly deployed in a 2 - dimensional square field $(100 m \times 100 m)$. The sensing range of each node is $9 m$, and 


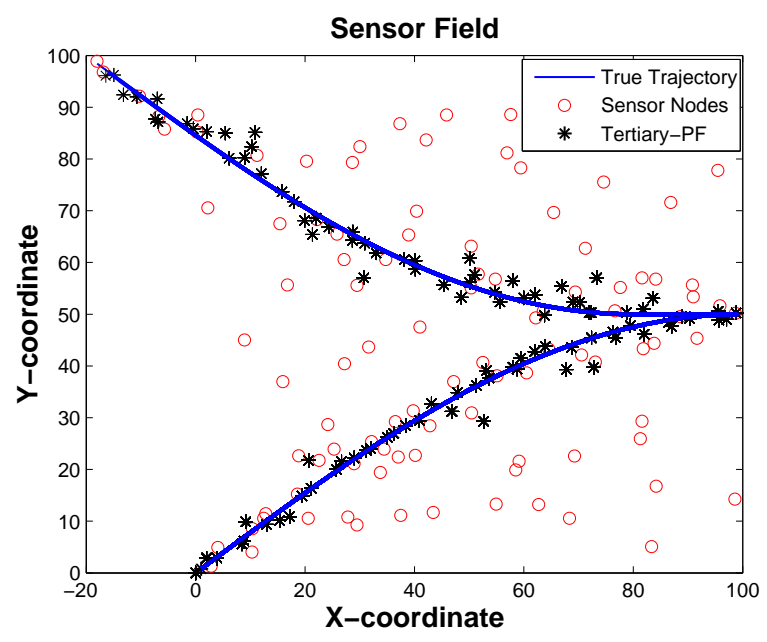

Fig. 3. Tertiary Particle Filtering

all $\beta_{j}$ are fixed to 1 , which means that all the sensor in the field have the same capability of sensing and communication. The parameters of the transition dynamical state model are set to the following values:

$$
\bar{\lambda}=\left[\begin{array}{cc}
1 / 625 & 0 \\
0 & 1 / 25
\end{array}\right], \quad \bar{n}=100, \quad \overline{\boldsymbol{S}}=\left[\begin{array}{cc}
10 & 0 \\
0 & 10
\end{array}\right]
$$

where the low mean state precision $\bar{\lambda}$ and the high degree of freedom $\bar{n}$ allow a general non informative prior. The performances of the BVF tracking algorithm are plotted in Fig. 1. Note the accuracy of the BVF to track the target, especially in the instant of abrupt change.

For the SOI-KF [8], despite the fact that the communication between sensors is reduced to one bit, it still requires sensing the distance information and comparing it to the predicted value. Instead of sensing the much more simple connectivity information required in BVF, it needs more complex sensing configuration of WSN. Furthermore, due to the linearization in the data processing phase, it cannot provide correct estimation in highly non-linear conditions, as shown in Fig. 2. At instant $t=50$, the target changes its direction and position immediately. Only by a long series of modifications that SOI-KF can catch the target.

For the PF in tertiary wireless sensor network [6]( See Fig. 3 ), the large amount of particles and their corresponding weights make it impossible to realize distributed signal processing. Furthermore, the dependence on the estimation of former instants leads to deviation in judgment of the target direction and may aggravate the error propagation at the same time, especially in the case of abrupt change in the target trajectory.

By employing GMM to approximate the distribution of particles at every instant, distributed signal processing can be achieved, while new problem of error propagation is involved. The information transferred between leaders is still

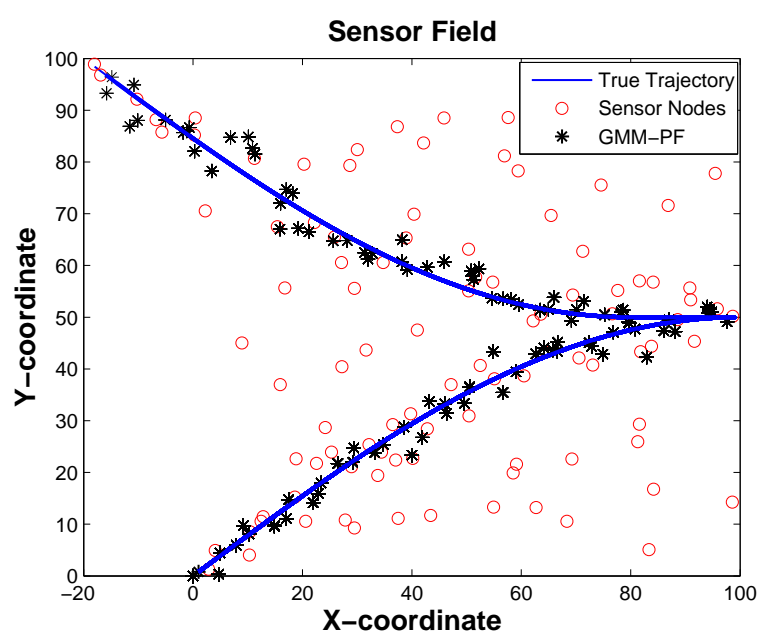

Fig. 4. GMM-PF estimation

more complex than that of BVF, since BVF transmits just one Gaussian statistic. Furthermore, it takes too much time to generate the GMM approximation of particles by KD-Tree[3] or EM $[5,11])$. The performance of this algorithm is shown in Fig. 4. In Fig. 6, we compare the MSE of the BVF and that of the GMM-PF.

\begin{tabular}{ccc}
\hline Algorithm & Mean of MSE & Max of MSE \\
\hline BVF & 1.8371 & 5.5928 \\
SOI-KF & 2.8125 & 20.4862 \\
Tertiary-PF & 1.8968 & 7.4431 \\
GMM-PF & 2.0274 & 5.6481 \\
\hline
\end{tabular}

Table 1. MSE comparison of the four algorithms

Fig. 5 and Fig. 6 show that under the same experimental conditions, the BVF algorithm succeeds in tracking the target with a more acceptable MSE at lower communication cost. Thanks to the distributed signal processing, the energy consumption is distributed among the region covered by the target, prolonging the lifetime of the WSN. Table. 1 compares the average and the maximum MSE of the different target tracking algorithms.

\section{CONCLUSION}

A binary variational filtering algorithm is proposed in the context of collaborative wireless sensor networks. The key points of the proposed algorithm are (i) the implicit compression of the filtering distribution (ii) the distributed implementation (iii) the lossless encoding by sending only Gaussian statistics between leader nodes and (iv) the binary quantification of the measured energy avoiding distance measure on the nodes. 


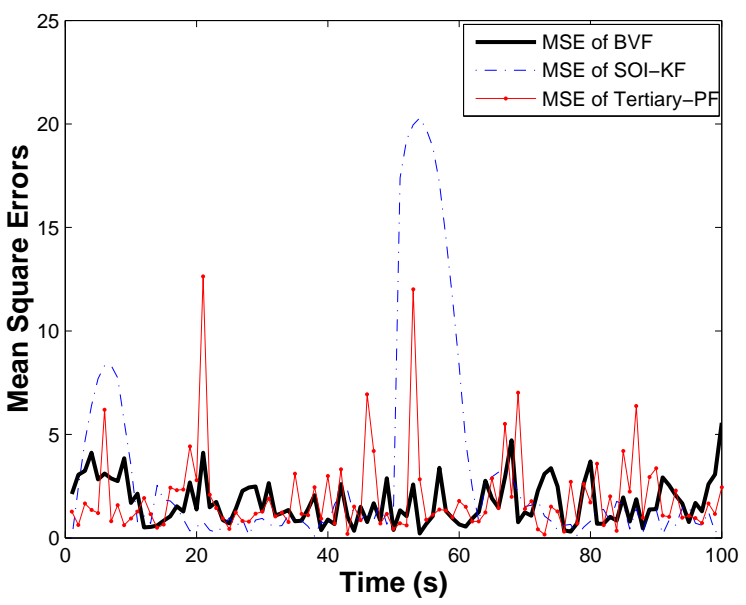

Fig. 5. MSE comparison of BVF, SOI-KF and Tertiary-PF.

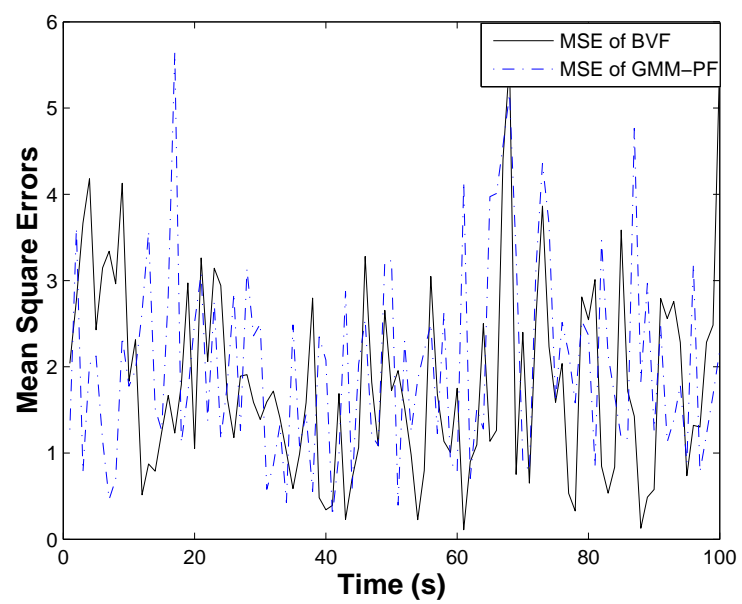

Fig. 6. MSE comparison of BVF and GMM-PF.

\section{REFERENCES}

[1] F. Zhao, J. Shin, and J. Reich, "Information-driven dynamic sensor collaboration for tracking applications," IEEE Signal Processing Magazine, vol. 19, no. 2, pp. 61-72, 2002.

[2] A. Doucet, B. Vo, C. Andrieu, and M. Davy, "Particle filtering for multitarget tracking and sensor management," in Proc. Int. Conf. Inform. Fusion, Mar. 2002, vol. 1, pp. 474-481.

[3] A. Ihler, J.W. Fisher III, and A.S. Willsky, "Particle filtering under communications constraints," in Proc. Statistical Signal Processing (SSP) 2005, 2005.

[4] Tom Vercauteren, Dong Guo, and Xiaodong Wang, "Joint multiple target tracking and classification in collaborative sensor networks," IEEE Journal on Selected Areas in Communications, vol. 23, no. 4, pp. 714-723, 42005.

[5] X. Sheng, Y.H. Hu, and P. Ramanathan, "Distributed particle filter with GMM approximation for multiple targets localiza- tion and tracking ion wireless sensor network," in Information Processing in Sensor Networks, 2005. IPSN 2005, Apr. 2005, pp. 181-188.

[6] Petar Djuric, Mahesh Vemula, and Mónica Bugallo, "Tracking with particle filtering in tertiary wireless sensor networks," in ICASSP, Philadelphia, PA, USA, March 18-23 2005.

[7] Hichem Snoussi and Cédric Richard, "Ensemble learning online filtering in wireless sensor networks," in IEEE ICCS International Conference on Communications Systems, Singapore, October 2006.

[8] Alejandro Ribeiro, Georgios Giannakis, and Stergios Roumeliotis, "Soi-kf: Distributed kalman filtering with low-cost communications using the sign of innovations," in ICASSP, Toulouse, France, 2006.

[9] A. Doucet, S. Godsill, and C. Andrieu, "On sequential Monte Carlo sampling methods for Bayesian filtering," Statistics and Computing, vol. 10, no. 3, pp. 197-208, 2000.

[10] J. Vermaak, N.D. Lawrence, and P Perez, "Variational inference for visual tracking," in Conf. Computer Vision and Pattern Recog, CVPR'03, June 2003.

[11] Verbeek, Jakob, Nunnink, Jan, Vlassis, and Nikos, "Accelerated em-based clustering of large data sets," Data Mining and Knowledge Discovery, vol. 13, no. 3, pp. 291-307, November 2006. 